\title{
Sticky-Particle Simulations of Barred Galaxies
}

\author{
Heikki Salo ${ }^{1}$, E. Laurikainen ${ }^{1}$, P. Rautiainen ${ }^{1}$, and R. Buta ${ }^{2}$ \\ ${ }^{1}$ Department of Physical Sciences, U. Oulu, Finland \\ email: heikki.salo@oulu.fi \\ ${ }^{2}$ Department of Physics and Astronomy, U. Alabama, USA
}

\begin{abstract}
We use sticky-particle simulations to estimate the bar pattern speeds in disk galaxies over a wide range of Hubble types. For early types our results agree with the fast rotation found in previous kinematical measurements, while for later types significantly slower bars are found. Slow bars tend to be small, and favor less luminous galaxies, for a given bulge-to-total ratio.
\end{abstract}

Keywords. galaxies: bars, galaxies: kinematics and dynamics, galaxies: evolution

\section{Introduction}

Pattern speed measurements with the Tremaine-Weinberg method favor fast bars, seemingly in contrast with live halo N-body models (see Athanassoula 2005, CeMDA 91, 9) which suggest a slowdown of bars via angular momentum exchange with massive halos. However, reliable direct measurements, using stellar absorption line kinematics, are available only for early-type spirals. To allow a wider comparison, we perform stickyparticle simulations utilizing gravitational potentials derived from near IR observations. This approach does not address the pattern speed evolution, but instead gives an estimate for the instantaneous pattern speed consistent with the observed morphology. We have recently applied this method to modeling of 38 galaxies from the OSUBGS survey, and are currently performing similar studies to the NIRSOS sample (see Laurikainen et al., this conference). This work is supported by the Academy of Finland.
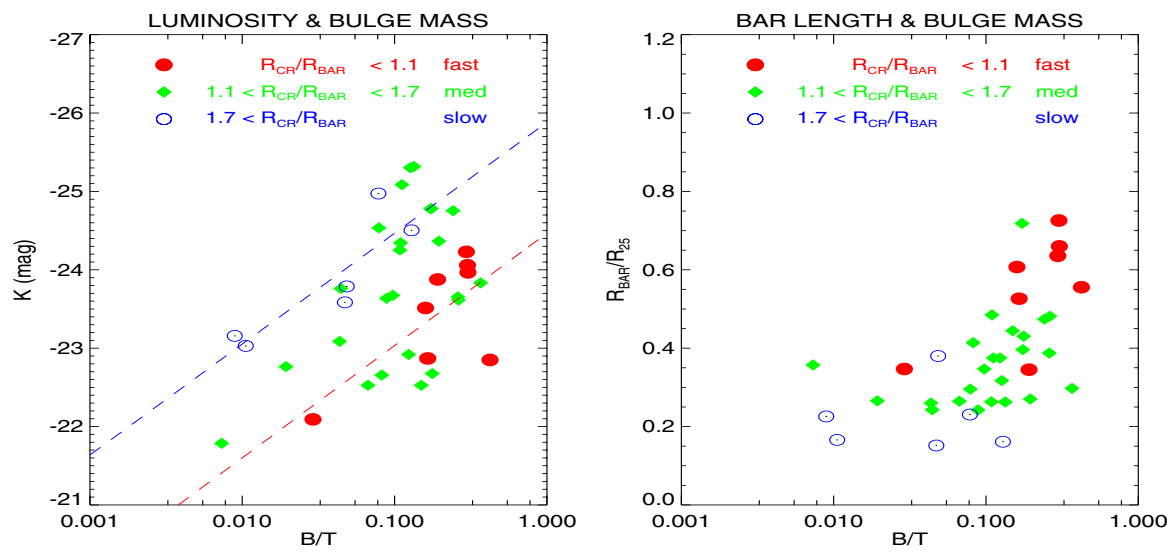

Figure 1. The location of galaxies with fast/intermediate/slow bars (measured in terms of bar length and corotation radius) in a $B / T$ vs. $K$, and $B / T$ vs $R_{B A R} / R_{25}$ diagrams. Bulge-to-total light ratios $B / T$ are from our $2 \mathrm{D}$ multicomponent decompositions (Laurikainen, Salo \& Buta 2005, MNRAS, 362, 1319), and the bar pattern speeds from simulations in Rautiainen, Salo \& Laurikainen (2005, ApJ 631, L129), performed with the method of Salo et al. (1999, AJ 117, 792). 